\title{
Viabilidad de comunidades profesionales de aprendizaje en sistemas educativos de bajo desempeño
}

\section{Sergio Morales-Inga}

https://orcid.org/0000-0001-8007-164X Universidad Nacional Mayor de San Marcos, Perú

sergio.morales@unmsm.edu.pe

\section{Oswaldo Morales-Tristán}

https://orcid.org/oooo-0o02-5298-9733 ESAN Graduate School of Business, Perú omorales@esan.edu.pe

\section{Resumen}

Las comunidades profesionales de aprendizaje (CPA) constituyen una de las estrategias de desempeño escolar más importantes. Sin embargo, la mayoría de las escuelas en las que se han aplicado pertenecen a sistemas educativos de alto desempeño, como los de Japón, Corea de Sur, Finlandia o Singapur, lo que deja por fuera la realidad educativa de América Latina. Mediante un estudio exploratorio, basado en observación etnográfica y entrevistas semiestructuradas, esta investigación vincula teoría y práctica para discutir la aplicabilidad de las CPA en escuelas públicas pertenecientes a sistemas educativos de bajo desempeño. Como conclusión, se halló que los componentes principales de las CPA (estructuras organizativas flexibles, liderazgos distribuidos, valores compartidos y culturas escolares colaborativas) están presentes, pero son precarios y no soportan la implementación de la estrategia.

\section{Palabras clave (Fuente: tesauro de la Unesco)}

Comunidades de aprendizaje; estructura organizativa; liderazgo; organización escolar; Perú. 


\title{
Viability of Professional Learning Communities in Low-Performance Educational Systems
}

\begin{abstract}
Professional learning communities (PLCS) are one of the most significant school performance strategies. Yet, most of the schools that have applied them belong to highperformance educational systems, such as those of Japan, South Korea, Finland or Singapore, leaving out the educational reality of Latin America. Through an exploratory study based on ethnographic observation and semi-structured interviews, this research links theory to practice to discuss the applicability of PLC to public schools in low-performance educational systems. In conclusion, it was found that the main components of PLCS (flexible organizational structures, distributed leadership, shared values, and collaborative school cultures) are present but precarious and do not support the implementation of the strategy.
\end{abstract}

Keywords (Source: Unesco thesaurus)

Learning communities; organizational structure; leadership; school organization; Peru. 


\title{
Viabilidade de comunidades de aprendizagem profissional em sistemas educativos de baixo desempenho
}

\author{
Resumo
}

As comunidades de aprendizagem profissional (CAP) constituem uma das estratégias de desempenho escolar mais importantes. Contudo, a maioria das escolas em que são aplicadas pertence a sistemas educativos de alto desempenho, como os do Japão, da Coreia do Sul, da Finlândia ou de Singapura, o que deixa por fora a realidade educativa da América Latina. Mediante um estudo exploratório, baseado em observação etnográfica e entrevistas semiestruturadas, esta pesquisa vincula teoria e prática para discutir a aplicabilidade das CAP em escolas públicas pertencentes a sistemas educativos de baixo desempenho. Como conclusão, verificou-se que os componentes principais das CAP (estruturas organizadas flexiveis, lideranças distribuidas, valores compartilhados e culturas escolares colaborativas) estão presentes, mas são precários, e não apoiam a implantação da estratégia.

Palavras-chave (Fonte: tesauro da Unesco)

Comunidades de aprendizagem; organização escolar; estrutura organizada; estrutura administrativa; liderança; Peru. 


\section{Aprendizaje organizacional y comunidades profesionales de aprendizaje}

Consideradas por DuFour como una "nueva y poderosa forma de trabajo conjunto que afecta profundamente las prácticas de gestión escolar" (2004, p. 11), las comunidades profesionales de aprendizaje (CPA) constituyen una de las estrategias de mejoramiento educativo más discutidas actualmente'. Teóricamente, sus orígenes advienen de la gestión empresarial, gracias a los trabajos de Argyris, Senge, Schön, Leithwood y Seashore en el campo del aprendizaje organizacional, un enfoque de gestión centrado en la creación reflexiva de conocimiento (Argote, 2011). Gracias a sus buenos resultados, no tardó mucho tiempo en trasladarse a la gestión educativa (Álvarez, 2006; Vescio, Ross y Adams, 2008; Mulford y Silins, 2010) como una de las estrategias de aprendizaje organizacional más discutidas. En pleno cambio de paradigma pedagógico, de uno individualista y jerárquico hacia uno colaborativo y reflexivo, las CPA encontrarían en las escuelas sitio ideal para encarnar espíritus de reforma (Sai y Siraj, 2015; Olivier y Huffman, 2016). Hoy por hoy, sus destacables resultados en mejoramiento del desempeño docente y estudiantil (Sigurðardóttir, 2010) pueden ser constatados en escuelas de diversos países, como Estados Unidos, Noruega, Finlandia, Nueva Zelanda, China, Hong Kong, Japón, Corea del Sur, Taiwán o Singapur². Es precisamente dicha evidencia la que, a pesar de ciertos cuestionamientos (DuFour, 2007), defiende su importancia y establece un consenso sobre sus cuatro componentes básicos: estructuras organizativas flexibles, liderazgo distribuido, valores compartidos y cultura escolar colaborativa.

\footnotetext{
Para una revisión extensa, véase Morales y Morales (2018).

2 Véanse: Cherrington y Thornton (2015); Tam (2015a, 2015b); Chen, Lee, Lin y Zhang (2016); Cheng y Wu (2016); Gutierez (2016); Higgins (2016); Lee y Kim (2016); Olivier y Huffman (2016); Pang, Wang y Leung (2016); Popp y Goldman (2016); Salleh (2016); Sjoer y Meirink (2016); Svanbjörnsdóttir, Macdonald y Frímannsson (2016a, 2016b); Vanblaere y Devos (2016, 2018); Wang (2016); Zhang y Pang (2016); Ahn (2017); Bellibas, Bulut y Gedik (2017); Dogan, Tatık y Yurtseven (2017); Oiao, Yu y Zhang (2017); Wennergren y Blossing (2017); y Zhang, Yuan y Yu (2017).
}

Una comunidad de aprendizaje profesional consiste en un grupo de profesionales que comparten objetivos y propósitos en común, que constantemente obtienen nuevos conocimientos a través de la interacción y que apuntan a mejorar la práctica. Es un ciclo donde el aprendizaje normalmente está integrado en el trabajo diario; los maestros adquieren nuevos conocimientos, lo prueban en la práctica y, a partir de la experiencia, obtienen aún más conocimiento. Lo hacen interactuando entre ellos, trabajando en colaboración. Este ciclo está fuertemente influenciado por:factores estructurales, que pueden fomentar la colaboración o dificultarla; factores culturales, que son creencias y valores de las personas; $y$ el estilo de liderazgo, que afecta en gran medida tanto a la cultura dentro de la escuela como a la estructura. Todos estos factores o caracteristicas están incrustados entre si y son interdependientes. (Sigurðardóttir, 2010, p. 397)

No obstante, la mayoría de escuelas donde dicha estrategia ha sido implementada forman parte de sistemas educativos de alto desempeño a nivel mundial (en países como Corea del Sur, Japón, Singapur o Finlandia). Ello significa que la teoría de las CPA se ha edificado recogiendo las características específicas de tales sistemas e ignora si podrían generar los mismos resultados en escuelas pertenecientes a otros sistemas parcial o totalmente distintos. Considerando que elementos como cultura escolar o estructura organizativa dependen de diversos contextos socioculturales y, además, determinan el funcionamiento de las CPA, discutir su aplicabilidad en contextos particulares constituye no solo un problema fundamental, sino también un paso previo.

\section{Bajo desempeño del sistema educativo peruano}

Según la Ley General de Educación 28044 (LGE), el objetivo del sistema educativo peruano (SEP) es desarrollar la institución educativa (IE) o escuela como una comunidad de aprendizaje cuyos objetivos prin- 
cipales sean: fortalecer su autonomía pedagógica y administrativa, desarrollar liderazgos democráticos, promover la participación de la comunidad educativa e incentivar una cultura de autoevaluación (Ministerio de Educación, 2003). Sin embargo, gestionar la intrínseca complejidad del SEP, debido mayormente a la diversidad sociocultural del territorio nacional, es un reto en términos de gestión.

En las últimas décadas, en el sistema educativo peruano han ocurrido una serie de importantes procesos que han determinado la estructura y el desempeño del sector educativo. [...] el estancamiento de la inversión en el sector educativo, la implantación de reformas normativas relacionadas con la docencia, tres cambios curriculares en una década, la ampliación de la cobertura de la educación básica y la reducción de la calidad educativa observada por medio de indicadores de respuesta (p. e. los logros de aprendizaje, tasas de deserción, retornos de la educación, niveles educativos alcanzados), entre otros. (Jopen, Gómez y Olivera, 2014, p. 1)

La educación en el país tiene un sinnúmero de necesidades y demandas, las mismas que nos plantean retos y desafios para afrontarlas de manera coherente y efectiva. Sin embargo, la prioridad inminente es impulsar el cambio que requieren los procesos educativos para la transformación efectiva de la escuela, en su dinámica, estructura y organización, con el fin de lograr resultados de aprendizaje significativos en los estudiantes y la sociedad. (Ministerio de Educación, 2015a, p. 10)

Como tal, el SEP se organiza en Educación Básica y Educación Superior. La básica (dividida en los niveles inicial, primario y secundario) tiene por objetivo brindar al estudiante una formación integral cimentada en capacidades que posibiliten su aprendizaje en los campos científico, artístico, cultural o deportivo. Asimismo, se divide en distintas modalidades, como Educación Básica Regular, dirigida a la mayoría de la población estudiantil; Educación Básica Alternativa, destinada al desarrollo de capacidades laborales; Educación Básica Especial, designada a estudiantes con discapacidad o talentos específicos; Educación Intercultural-Bilingüe, asignada a población nativa; Educación Técnico-Productiva, enfocada en el desarrollo de competencias laborales; $y$ Educación Comunitaria, ejercida por organizaciones no educativas. Precisamente en razón de dicha complejidad, el SEP muestra limitaciones que generan su bajo desempeño (Jopen et al., 2014) e impactan negativamente en la sociedad. Según Beltrán y Seinfeld, "la persistencia de la baja calidad educativa [...] se ha convertido en uno de los principales problemas sociales del país, con dramáticas consecuencias en el largo plazo" (2013, p. 9).

Algunas de estas limitaciones, explicadas con profundidad en literatura especializada (Beltrán y Seinfeld, 2013; Guadalupe, León, Rodríguez y Vargas, 2017), son: la carencia de descentralización educativa, requerida como política de Estado y sinónimo de democratización de la educación (Ministerio de Educación, 2013); la falta de inclusión educativa, referida a la Educación Intercultural-Bilingüe y a la Educación Básica Especial (OCDE, 2016); la desvalorización docente, generada por limitadas condiciones laborales y carencias en su formación profesional (Díaz, 2015) -ante lo cual se promueven estrategias de revalorización, como remuneración meritocrática, bonos de atracción o reconocimiento a la trayectoria (Cuenca, 2017; Ministerio de Educación, 2016a)-; los problemas de inversión y gasto, los cuales repercuten en la calidad educativa, la gratuidad de la enseñanza, el salario docente y el desarrollo de infraestructura (Jopen et al., 2014; OCDE, 2016); el bajo desempeño estudiantil, el cual compone una variable importante del problema de la educación nacional (Moreano, 2017); y los contenidos curriculares, ya que la modificación del último Currículo Nacional de Educación Básica (Alayo, 2017; Ministerio de Educación, 2017), realizada en medio de procesos de reforma (Chuquilin y Zagaceta, 2017), ha mostrado injerencia en el desempeño 
del Ministerio de Educación (El Comercio, 2017a) y de las escuelas (El Comercio, 2017b), así como en la ejecución de políticas educativas (La República, 2017).

Para remediar dicho escenario, organismos competentes han desarrollado el modelo de gestión escolar centrado en aprendizajes, el cual se compone de ocho prácticas o compromisos (Ministerio de Educación, 2015b). Asimismo, se han establecido pautas de buen desempeño, tanto docente -para "transitar de la enseñanza tradicional a la producción del conocimiento" (Ministerio de Educación, 2012, p. 8)- como directivo, cuyos pilares son la gestión orientada a las personas, a la obtención de resultados y al liderazgo pedagógico (Ministerio de Educación, 2015b). A ello se suman el Proyecto Educativo Nacional al 2021 (Consejo Nacional de Educación, 2006) y Semáforo Escuela, un mecanismo de supervisión gestionado por la Oficina de Seguimiento y Evaluación Estratégica del Ministerio de Educación, cuya finalidad es supervisar las escuelas del país y optimizar su gestión mediante el levantamiento de indicadores de desempeño, horas efectivas de clase, récord de asistencia o necesidades de infraestructura (Ministerio de Educación, 2016b).

Dado el complejo contexto sociocultural peruano, el SEP se compone de un sistema privado (Villalobos, 2017) y uno público, compuesto este no solo por escuelas polidocentes, sino también por múltiples modelos de instituciones educativas: monolingües, cuyas lecciones se dictan en idioma único; intercultural-bilingüe, donde se emplea la lengua nativa de la comunidad; multiedad, donde un docente se encarga de múltiples secciones; multigrado, donde más de un docente tiene bajo su cargo hasta seis grados; unidocente, donde un único docente desempeña también funciones directivas; polidocente multigrado, donde cada docente se encarga de dos secciones; y polidocente completa, donde diversos docentes imparten clases a una sola sección (Ministerio de Educación, 2016b).

\section{Institución educativa Villa Magisterial}

Para la Ley General de Educación, la escuela constituye la "primera y principal instancia descentralizada" del sistema educativo peruano, al tener como finalidad la formación integral de los estudiantes mediante el desarrollo del Proyecto Educativo Institucional y el establecimiento de vínculos de cooperación con padres de familia y gobiernos locales (Ministerio de Educación, 2003). No obstante, su situación y desempeño varían según los diversos contextos socioeconómicos y culturales en los que se sitúan, pues "la mayoría de escuelas tienen carencias y presentan dificultades que se manifiestan en el escaso logro de aprendizajes de sus estudiantes y en la contribución mínima en su formación integral" (Ministerio de Educación, 2015a, p. 10). Esta complejidad genera que la mayor diversidad organizacional existente dentro del SEP recaiga principalmente sobre las escuelas, de ahí la necesidad de reconsiderarlas como unidades de análisis en relación con el desempeño escolar.

La institución educativa Villa Magisterial (VM) es una escuela pública compuesta por 12 profesionales (10 docentes, 1 auxiliar y 1 directora) y aproximadamente 200 estudiantes en sus niveles inicial y primario. Fue fundada el año 2009 y se ubica en la sierra del Perú, ciudad de Arequipa, a más de 2200 m.s.n.m. Como institución pública, VM presenta carencias de personal docente (reciben apoyo de la organización no gubernamental Enseña Perú, que deriva docentes a zonas vulnerables), administrativo (carecen de subdirección, coordinación académica y secretaría), de seguridad (reciben apoyo de padres de familia) y cocina (reciben apoyo del Programa Nacional de Alimentación Escolar Oali Warma del Ministerio de Desarrollo e Inclusión Social), así como también de infraestructura (falta de aulas, servicios básicos y bienes muebles, como pizarras, estantes y escritorios). Según Beltrán y Seinfeld, "las variables vinculadas con la infraestructura y la gestión de la educación son determinantes fundamentales en el desempeño escolar de los colegios; en algunos 
casos, incluso, por encima de las características socioeconómicas del estudiante" (2013, p. 12). A esta situación se agrega la impuntualidad y baja participación docente que impiden el correcto desempeño de la escuela y generan un clima laboral nocivo.

Sin embargo, como parte de un conjunto de políticas comandadas por el Ministerio de Educación (Salazar, 2017), se ejecutaron una serie de medidas que tuvieron como actores principales a los docentes de VM: nombrar nueva dirección, desplazar el inicio de clases por 30 minutos (para brindar oportunidades de puntualidad a docentes que vivían a horas de distancia), convocar con regularidad reuniones de coordinación para permitir el diálogo constante, admitir la participación docente en la gestión educativa para asumir su liderazgo, brindar acceso a los padres de familia como garantes del desempeño de docentes y estudiantes, facultar la retroalimentación pedagógica para generar que docentes aprendan diversas estrategias pedagógicas de sus colegas y ejecutar distintos proyectos ${ }^{3}$ que generen un sentido de responsabilidad colectiva. Luego de dos años, tales prácticas reformaron el desempeño de VM y se produjeron importantes cambios que impactaron visiblemente en el trabajo pedagógico, pues los docentes empezaron a sentirse parte de la escuela al ser partícipes directos de su gestión. En palabras de Cadena y Orcasitas, "el aula es un lugar útil a la vida humana ya que da sentido a los grupos de pertenencia, favoreciendo el trabajo cooperativo desde aprendizajes creativos y adecuados que será evaluado por el sistema educativo" (2016, p. 383).

\section{Metodología}

Vinculando teoría y práctica, el presente estudio de caso exploratorio tiene por objetivo discutir

3 Como "Ecoescuela" (2015-2016), que consistió en sembrar pequeños árboles dentro y alrededor de la IE (ubicada en zona desértica) para promover la gestión ambiental, o "Mejoremos la educación en nuestro Perú" (2015), que consistió en visitas a diversas escuelas para concientizar sobre los problemas de la educación nacional mediante exposiciones realizadas por los mismos estudiantes. la aplicabilidad de las CPA en escuelas públicas pertenecientes a sistemas educativos de bajo desempeño, tomando la institución educativa Villa Magisterial como referente empírico. Para ello, se analizan los componentes principales de las CPA (estructura organizativa flexible, liderazgo distribuido, valores compartidos y cultura escolar colaborativa), en función de la realidad de Villa Magisterial, con la finalidad de determinar si tales componentes pueden ser hallados en una escuela pública perteneciente a un sistema educativo de bajo desempeño. El estudio emplea técnicas como la observación etnográfica, usualmente aplicada en ámbitos educativos (Yon, 2003) a fin de comprender las escuelas "por dentro" (Woods, 1987), para analizar la interacción de los distintos componentes organizacionales de la escuela, y entrevistas semiestructuradas a docentes y directivos, para aprehender su perspectiva de las cosas.

Se destaca que el referente empírico considerado difiere de los usualmente utilizados por estos estudios -los cuales aluden a sistemas educativos de países como Finlandia, Singapur o Corea del Sur, destacables a nivel mundial pero diferentes de la realidad educacional de América Latina (Martins, 2016)-, por lo que la presente investigación busca subsanar un vacío en la teoría de las CPA, ya advertido en relación con sistemas educativos no occidentales, aunque de alto desempeño (Wong, 2010; Pang y Wang, 2016; Oiao et al., 2017; Zhang et al., 2017). Apelando a un estudio de caso exploratorio -un diseño común en investigación educativa (Álvarez y San Fabián, 2012)- sustentado teóricamente, se busca compensar el ateoricismo que afecta dicho diseño (Rule y John, 2015). Asimismo, dado que las características del referente empírico impiden su tratamiento cuantitativo (generalmente empleado en estudios sobre (PA), se usa un enfoque principalmente cualitativo que, aunque minoritario en este tipo de estudios (Zhang et al., 2017), sea capaz de aprehender las cualidades organizativas del referente empírico (Sandín, 2003). Por último, como finalidad, el estudio busca fomentar la discusión del 
empleo de estrategias organizacionales dirigidas al elemento más importante de todo sistema educativo: la escuela.

\section{Importancia de la estructura organizativa: entre homogeneidad y heterogeneidad}

$\mathrm{El}$ primer y más fundamental componente de cualquier organización es su estructura, dada la influencia que ejerce sobre el desempeño (Hunter, 2015). Considerando que las escuelas son formas de organización, la estructura también se halla muy vinculada al mejoramiento educativo (Lam, 2005). Asimismo, dado que las CPA tienen como finalidad optimizar el desempeño educativo, su relación con la estructura organizativa es también fundamental. Al respecto, múltiple evidencia señala que, para su constitución, las CPA necesitan de estructuras organizativas flexibles capaces de permitir la retroalimentación entre sus diversos niveles (Gray, Kruse y Tarter, 2015; Kruse y Johnson, 2017). Caso contrario, una estructura organizativa rígida podría impedir su óptimo desempeño (Harris y Jones, 2010). La educación pública, sin embargo, no reconoce debidamente la trascendencia de dicho componente.

El SEP distingue dos grandes dominios: la comunidad educativa, compuesta por estudiantes, padres de familia, profesores, directivos, exalumnos y líderes comunales próximos a la IE, y las instancias de gestión educativa, representadas por organismos como el Ministerio de Educación (Minedu), las Direcciones Regionales de Educación (DRE), las Unidades de Gestión Educativa Local (UGEL) y las diversas instituciones educativas. Considerando esta distinción, la estructura organizativa de Villa Magisterial (véase Figura 1) puede ser comprendida en cuatro niveles fundamentales:

a. Nivel gubernamental, compuesto por: el Ministerio de Educación, órgano rector de la política educativa, encargado de elaborar el Proyecto Educativo Nacional y los diseños curriculares, dirigir el Programa Nacional de Formación y
Capacitación Permanente del magisterio y el Programa Nacional de Investigación Educativa, además de afianzar el presupuesto nacional de educación y evaluar los aprendizajes mediante el Sistema Nacional de Evaluación, Acreditación y Certificación de la Calidad Educativa; las DRE, responsables de brindar el servicio educativo en distintas regiones del país, autorizar el funcionamiento de IE públicas y privadas, formular y evaluar el presupuesto educativo en las regiones e identificar prioridades de inversión en infraestructura educativa; y las UGEL, instancias autónomas, descentralizadas y provinciales encargadas de desarrollar las capacidades de gestión pedagógica y administrativa de las IE en pro de su autonomía.

b. Nivel directivo, conformado por: la dirección -según la LGE-, máxima autoridad y representante legal de la IE, encargada de la gestión pedagógica y administrativa, de evaluar y asesorar el desempeño de la IE, de supervisar y coordinar el trabajo docente, de promover una práctica de evaluación y autoevaluación, así como de prevenir y/o solucionar conflictos organizacionales; y los puestos administrativos vinculados - para el caso de VM, este nivel se encuentra compuesto únicamente por la directora, al no contar con áreas de subdirección, coordinación académica o secretaría.

c. Nivel participativo, compuesto por tres organizaciones con capacidad de participación en la gestión de la IE: las Asociaciones de Padres de Familia, encargadas de supervisar el manejo adecuado del material pedagógico e infraestructura, participar en la formulación y ejecución del Proyecto Educativo Institucional y del Plan Anual de Trabajo, así como en el comité de evaluación para el ingreso, ascenso y permanencia de personal administrativo y/o docente; el Consejo Educativo Institucional, organismo de concertación ciudadana presidido por la dirección de la IE y conformado por padres de 
familia, profesores, alumnos, exalumnos y personal administrativo, cuya finalidad es participar, evaluar y vigilar la gestión educativa; y el profesorado, conformado por los docentes de la IE, quienes participan en la gestión mediante los comités.

d. Nivel ejecutivo, compuesto por equipos de trabajo de profesores y estudiantes encargados de la ejecución de estrategias producidas en los niveles superiores.

\section{Figura 1. Estructura organizativa de Villa} Magisterial

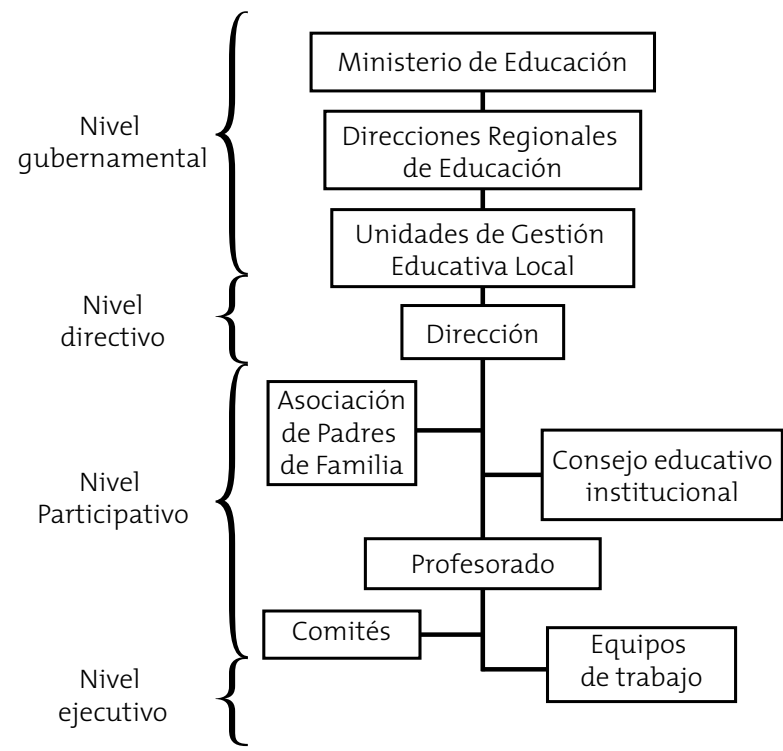

Fuente: elaboración propia.

En el SEP, las instancias de gestión (nivel gubernamental) gozan de cierta estabilidad, pues permanecen homogéneas prácticamente a nivel nacional. La diversidad organizacional empieza recién en los niveles directivo, participativo y ejecutivo (cuya conformación es llamada comunidad educativa), ya que ellos comprenden las múltiples escuelas cuyas características y necesidades varían según los distintos contextos socioculturales. Esta diversidad organizacional comporta mayor complejidad en la gestión escolar, al producir estructuras escolares heterodoxas para cada escuela (unidocente, multigrado, polidocente, etc.), las cuales generan brechas y desigualdades en los aprendizajes (Guadalupe et al., 2017, p. 179-208). Según Beltrán y Seinfeld, "son las zonas rurales las que tienen mayores restricciones para brindar un servicio educativo de calidad así como menor gasto público destinado a la educación" (2013, p. 121). Comprender la importancia de dichas estructuras en la gestión escolar y reconocer su heterogeneidad son condiciones necesarias en la discusión sobre el establecimiento de CPA. El SEP debe reconsiderar las estructuras organizativas no como una simple representación gráfica del esqueleto de una escuela, sino como un elemento determinante de su desempeño, que podría no funcionar correctamente al estar sometido a la influencia de la diversidad sociocultural.

\section{Dilemas del liderazgo escolar: doble función directiva y comités de gestión}

Como atributo organizacional, el liderazgo no solo es posibilitado por la estructura organizativa (Rutherford, 2006), sino que además guarda un vínculo esencial con el mejoramiento educativo (DiPaola y Hoy, 2015). En referencia a las CPA, el liderazgo asume un rol fundamental, al constituir uno de sus componentes centrales (Buttram y Farley-Ripple, 2016; Chen et al., 2016; Vanblaere y Devos, 2016, 2018; Wang, 2016). Según Pang et al., "para crear, desarrollar y sostener una comunidad profesional de aprendizaje en una escuela, se necesita activar el liderazgo en todos los niveles" (2016, p. 233). Dada la naturaleza del cargo, se considera que la única instancia capaz de ejercer el liderazgo escolar es la dirección. Sin embargo, uno de los problemas más urgentes a los que se enfrenta la función directiva en el SEP es la sobrecarga laboral, por la doble función que realizan los directores: gestionar y administrar. Determinar la naturaleza del cargo directivo constituye un punto importante a considerar antes de establecer CPA.

La evidencia, tanto nacional como internacional, revela que cuando el director de una escuela enfoca las tareas con liderazgo pedagógico, [...] su actuar tiene un impacto positivo en el 
rendimiento académico de los estudiantes. En nuestro pais, la mayoría de las funciones que se atribuyen por ley a los directores son de carácter administrativo, lo que no promueve este tipo de liderazgo. Esta situación se ve agravada por la forma de selección o designación, la situación laboral inestable, la diversidad de estructuras y tipos de escuelas, la ausencia de propuestas de formación y capacitación, la simultaneidad de cargos y funciones docente-director, y la ausencia del liderazgo pedagógico en su desempeño. (Ministerio de Educación, 2015a, p. 16)

La dirección es un cargo complejo, porque requiere de muchas competencias en lo administrativo y pedagógico. Por un lado, tienes que gestionar que la escuela marche como un reloj-asistencia, puntualidad, documentación, infraestructura, coordinación con actores externos, etcétera- $y$, por otro lado, tienes todo el trabajo pedagógico de seguimiento y acompañamiento. Respecto a este punto, considero que, al haber estudiado la carrera docente, los directores tienen los conocimientos y experiencia necesarios para saber lo que se espera de un buen docente. Sin embargo, creo que la mayoría de esfuerzos para fortalecer a los directores debería dirigirse a sus competencias de gestión. Hay tantas decisiones por tomar que su trabajo se vuelve abrumador. No creo que el gerente general de una gran empresa tenga las mismas demandas que tiene un director de escuela. (Profesora de Computación; comunicación personal)

De todos los estilos de liderazgo existentes en la literatura, uno es imprescindible para la conformación de CPA: el liderazgo distribuido o compartido, el cual encarna uno de sus componentes esenciales (Castillo, Puigdellivol y Antúnez, 2017). Como tal, "el liderazgo compartido reconoce que diferentes personas -no solo las que ocupan cargos oficiales-pueden emerger como fuentes de influencia en diferentes ocasiones, con efectos positivos" (Bolivar, 2011, p. 266). Según Leithwood, el liderazgo distribuido genera que la organización se beneficie de una mayor participación, al permitir a sus integrantes sumar sus capacidades individuales y promover "un mayor sentimiento de interdependencia y un sentido de cómo el propio actuar tiene un efecto sobre la organización en su conjunto" (2009, p. 61). Por ello, el liderazgo compartido "debería ser una característica prioritaria [...] de una comunidad profesional de aprendizaje efectiva" (Carpenter, 2015, p. 685). Asimismo, sólida evidencia demuestra resultados positivos cuando el profesor deja de ser un agente únicamente pedagógico para constituirse como un agente de gestión mediante la promoción del liderazgo docente (Bolívar, 2011; Watson, 2014; Lai y Cheung, 2015; Diamond y Spillane, 2016). ¿Cómo y mediante qué organismos se gesta el liderazgo docente en el SEP?

Según la LGE, el docente constituye un agente fundamental del proceso educativo, al tener como misión contribuir a la formación integral de los estudiantes y participar en la gestión de la IE. No obstante, su participación se realiza formalmente mediante los comités de gestión -órganos presididos por los directores, pero liderados por los docentes-, cuya finalidad es atender determinados aspectos de la escuela. En ellos, son los docentes quienes participan desarrollando estrategias, planes y proyectos, programando diversas actividades de su comité y supervisando no solo su propio trabajo o el de sus colegas, sino también la misma gestión escolar. Generalmente, los comités son los únicos mecanismos organizacionales formales que promueven el liderazgo docente y evitan la sobrecarga laboral de la dirección, al distribuir la gestión hacia personal no directivo. Dada su naturaleza departamental, existen diversos tipos de comité: de presupuesto, seguridad, policía escolar, Cruz Roja, Defensa Civil, medio ambiente, recursos propios (gestión de ingresos), académico (búsqueda de capacitaciones docentes), inventario (gestión de bienes muebles), apoyo informático (mantenimiento de equipos de cómputo), etcétera. Si bien el Minedu exige un número determinado de comités, hay espacio para la creación de otros según las necesidades específicas de cada escuela. 
Estamos bien organizados. Tratamos de ver todas nuestras necesidades y para ello hemos creado los comités, tanto los que nos pide el Ministerio como también los que necesitamos nosotros por propio contexto. Nos hemos repartido funciones viendo la habilidad de cada docente. Hay docentes que son hábiles en gestión y hay docentes que son más de acción. Esa es la organización que tenemos dentro de la institución; vemos, no imponemos, según el potencial que tenga cada docente y según su compromiso. (Profesora de nivel inicial: 3 años; comunicación personal)

De no haber comités, el profesor dejaría de ser participe de la organización. La distribución de comités es necesaria porque hay que ayudar a la dirección. (Profesora de nivel inicial: 5 años; comunicación personal)

En VM los comités mantienen una dinámica sencilla, pero funcional. Su labor se ejecuta en reuniones de coordinación demandadas por padres de familia y docentes. Una vez solicitadas, la directora autoriza el proceso, convoca a los docentes y dirige el diálogo, pero sin reducirlo a su estamento. En ellas, docentes, directora y padres de familia dialogan de forma equitativa; no obstante, son los docentes quienes asumen el liderazgo y la responsabilidad de solucionar dichas problemáticas. Así, los comités constituyen el mecanismo organizacional más importante del liderazgo docente y la gestión escolar. Sin embargo, al depender de las necesidades de cada escuela, también están sujetos a la influencia de la diversidad sociocultural. Asimismo, al depender del profesorado, sufren las carencias de docentes, quienes usualmente deben trabajar en más de un comité, lo cual genera sobrecarga laboral. A diferencia de ciertos sistemas educativos de alto desempeño, en VM (y escuelas semejantes) el liderazgo docente no se ejerce desde departamentos académicos sólidos, sino mediante comités que, en cuanto sea posible, sacan adelante la gestión de la escuela. En razón de lo expuesto, cualquier intento de establecer CPA debe considerar los dilemas de la doble función directiva y los comités de gestión.

\section{Dos valores organizacionales: visión y compromiso}

Los valores constituyen recursos esenciales para el óptimo funcionamiento de toda organización, especialmente en escuelas, dada su naturaleza pedagógica. Como tales, ellos componen parte importante de las CPA, sea integrando sus dinámicas (Chen et al., 2016; Qiao et al., 2017) o posibilitando su emergencia (Huffman, 2003; Tam, 2015b). Su importancia está en la influencia que ejercen en el comportamiento individual y organizacional, al vincularse a los objetivos de la institución. En términos de Pirela y Sánchez, "los valores se definen como principios rectores del comportamiento humano, pues deciden la actuación de las personas de una manera y no de otra" (2009, p. 180).

En el SEP, los valores, aunque no sean del todo reconocidos en sus efectos concretos, son comprendidos dentro de los compromisos que docentes y directivos deben seguir (Ministerio de Educación, 2012). En el caso de VM, los docentes entrevistados destacaron la presencia de distintas prácticas basadas en valores que influenciaban de forma positiva su comportamiento individual y su desempeño organizacional. Mediante prácticas de gestión (crítica constructiva, reflexión grupal o búsqueda conjunta de soluciones) o distintas labores de apoyo (ayuda económica mediante préstamos o actividades profondos, felicitaciones por cumplimiento de objetivos o preocupación por la salud de colegas), el equipo de VM promueve valores que cohesionan sus dinámicas, como contrapeso de sus limitaciones materiales.

La solidaridad, el respeto, también la puntualidad veo que se practican. La empatía también. Por ejemplo, estamos en una reunión de planificación, en los primeros días, y ahora, con esta situación, nos quejamos de la situación económica. A veces exageramos en préstamos; entonces 
nosotros tenemos que ayudarnos, tener consideración, y de repente con la realización de alguna actividad nos levantamos la moral. Esto es solidaridad, sentir empatía respecto del otro. (Profesor de primer grado; comunicación personal)

Hay mucho respeto. Se respiró eso desde el primer momento. Hay un tema de respeto, hay un tema de tolerancia, hay un tema de saber escuchar a los demás. Eso se puede percibir en los primeros cinco minutos de estar con ellos. (Profesor de sexto grado; comunicación personal)

Para serle sincero, en veintinueve años de servicio que tengo, este es el colegio donde he podido encontrar que verdaderamente se practican valores. Hay amistad entre colegas y compromiso de trabajo; hay entendimiento; hay un clima institucional extremadamente bueno, desde la dirección hasta padres de familia, alumnos y profesores. (Profesor de quinto grado; comunicación personal)

Sin embargo, a pesar del claro consenso, no existen políticas orientadas a la promoción institucional de valores. Pese a realizarse en su dimensión formal (en reuniones de coordinación o en los comités), los valores de VM encuentran su génesis en la dimensión informal, tal y como ocurre en organizaciones cuyas dinámicas informales impactan en su funcionamiento formal (González, 2003). En VM, los valores también se practican mediante formas de socialización ocurrentes en reuniones de coordinación y planificación (dimensión formal), pero, con mayor arraigo, en días festivos, como el día del maestro, o en los cumpleaños de los miembros (dimensión informal).

Todos nos apoyamos y respetamos. Eso siempre hubo desde que el colegio se inició, y siempre hemos dicho en la institución que, venga quien venga, debe ser bien recibido, darle buen trato y apoyarlo para trabajar todos en conjunto y en equipo, porque asi se sale adelante en cualquier sentido. Y esto es lo que hay en esta institución educativa. (Profesora de tercer grado; comunicación personal)

Aqui hay una socialización más informal, en espacios informales, lo cual es bueno, porque se genera directamente en esos espacios. Se generan, a partir de las reuniones que hemos tenido, en conversaciones abiertas, en espacios libres, directamente con los profesores. En mi anterior trabajo, era un tema un poco más estructurado, porque el director académico te llevaba profesor por profesor a presentarte. Te decia "este es tu horario de trabajo, esta es tu área, trabajas con estos grados, y estas son tus listas". (Profesor de sexto grado; comunicación personal)

El caso de los valores devela un punto crucial: aunque se trate del comportamiento de una organización, la instancia individual es fundamental. Dado que los valores organizacionales constituyen parte de las CPA, la importancia del individuo destaca, al ejercer los valores que la organización demande. En palabras de Álvarez, "toda organización que adopta el enfoque del aprendizaje organizacional se caracterizará [...] por considerar al individuo como el centro de la organización" (2006, p. 14). No obstante, las acciones individuales no impactarían, de no estar vinculadas a tópicos centrales para el cumplimiento de objetivos organizativos: la visión y el compromiso. En primer término, la visión conforma uno de los elementos de las CPA, ya que "nos hace pensar en una idea común en beneficio de toda la organización. Es como un faro, una guía que orienta las metas institucionales" (p. 25). En segundo término, el compromiso es necesario para la gestión educativa, dada su relación con el desempeño de las CPA, pues su auge o caída dependen "del elemento más importante en lo que refiere a mejoramiento del desempeño en cualquier escuela -el compromiso y la persistencia de sus educadores" (DuFour, 2004, p. 11). Según Ahn, "las iniciativas de reforma y participación docente necesitan operar sobre la base del voluntarismo para tornarse auténticas y sostenibles" (2017, p. 83). En el caso de VM, esto constituye un hecho palpable. 
Un tema súper relevante fue el de las relaciones cálidas entre toda la comunidad educati$v a$, desde la directora hasta los docentes. Había mucha preocupación por el otro, más allá de temas laborales. Compartíamos espacios informales que generaban vínculos afectivos. Y tener ese ambiente tan positivo también favorecía a tener un buen desempeño, confianza y apertura para aprender unos de otros a mejorar nuestra práctica pedagógica. Compartíamos la visión común de querer darles a nuestros estudiantes lo mejor. Aspirábamos a la excelencia, no importaba el trabajo, el esfuerzo o el tiempo que eso implique. (Profesora de computación; comunicación personal)

Todos los profesores estamos comprometidos en dar cada uno nuestro potencial en los diferentes comités. Tenemos una directora que es animosa, que está siempre con nosotros, con los padres y con los estudiantes. Yo lo veo; la organización anda por buen camino. Hay reconocimiento, hay gestión, hay preocupación de la administración por los docentes en hacer bien el trabajo educativo y para obtener logros más que regulares, logros positivos. (Profesor de primer grado; comunicación personal)

En VM, el compromiso individual es valorado porque coadyuva al compromiso organizacional. Por ello, todo el equipo se halla comprometido en la gestión mediante diversas prácticas: nulo ausentismo, cumplimiento de labor pedagógica, participación en la gestión, generación de un clima laboral óptimo o ejecución voluntaria de labores que excedan la propia función, de modo que, dada la carencia de personal, es normal encontrar docentes limpiando las aulas, reparando muebles averiados o asistiendo en la cocina. Aunque los valores que construyen una visión compartida se ejecuten en la dimensión informal, es necesario que su promoción y desarrollo se encuentren debidamente estipulados como elementos centrales de la gestión escolar. Ante la aplicabilidad de CPA, es necesario destacar los valo- res no como un accesorio de las interacciones, sino como su componente central.

\section{Cultura escolar colaborativa}

En referencia a la gestión educativa, la cultura escolar es trascendental (Deal y Peterson, 2016). Dado su carácter holístico, hablar de cultura escolar implica hablar de dinámica organizacional, pues comprende todo aquello que hace que determinada escuela sea lo que es. Según Peterson y Deal, la cultura escolar se encuentra en los elementos más profundos de toda escuela: "en las reglas y asunciones no escritas, en la combinación de rituales y tradiciones, en la formación de símbolos y artefactos, en el lenguaje y el fraseo específicos que docentes y estudiantes emplean, así como en las expectativas sobre el cambio y el aprendizaje que saturan el mundo de la escuela" (2009, p. 9). Para los autores, "la cultura influencia y forma las maneras en que docentes, estudiantes y administrativos piensan, sienten y actúan" (pp. 9-10), al constituir "una poderosa red de rituales y tradiciones, normas y valores que afectan cada rincón de la vida escolar" (p. 10). De ahí que consideren que "el término cultura provee una forma más precisa e intuitivamente atractiva de ayudar a los líderes escolares en el entendimiento de las reglas y tradiciones no escritas, costumbres y expectativas de su propia escuela" (Deal y Peterson, 2016, p. 7).

Teniendo en cuenta que las CPA refieren a la dinámica escolar y se vinculan con el mejoramiento educativo, es normal que se las relacione con la cultura escolar (Sai y Siraj, 2015; Olivier y Huffman, 2016; Bellibas et al., 2017). Dicho vínculo postula que las CPA muestran mayor posibilidad de desarrollo, si en la escuela existe una cultura escolar positiva. Caso contrario, si esta es rígida o muestra poca adaptabilidad frente al cambio, podría convertirse en un serio obstáculo (Zhang y Pang, 2016; Ahn, 2017). Son, precisamente, las culturas escolares colaborativas (Sutton y Shouse, 2016) las que representan ese tipo de cultura flexible y apta para el establecimiento 
de CPA. Según fuerte evidencia, una cultura escolar colaborativa influye en el desarrollo de CPA (Buttram y Farley-Ripple, 2016; Chen et al., 2016; Popp y Goldman, 2016; Salleh, 2016; Sjoer y Meirink, 2016; Svanbjörnsdóttir et al., 2016b; Qiao et al., 2017), siendo incluso capaz de lograr que una escuela de bajo performance obtenga un alto desempeño (Ahn, 2017) o posibilitar el primer paso de una reforma del sistema educativo (Harris y Jones, 2010). Según Pang y Wang, "colaborar con los colegas es una manifestación de una comunidad profesional fuerte que mostrará un gran impacto en el desarrollo de un sentido de responsabilidad colectiva en el aprendizaje estudiantil" (2016, p. 194). Se puede afirmar, a este respecto, que una cultura escolar colaborativa es el cimiento de las CPA.

En relación con nuestro referente empírico, $\mathrm{VM}$ destaca por su trabajo colaborativo y un claro matiz pedagógico para con los mismos docentes, quienes observan constructivamente las estrategias de enseñanza de sus colegas y establecen algo cercano a una cultura de aprendizaje. Tanto la estructura organizativa (que, dentro de sus límites, posibilita el trabajo de los comités) como la influencia de los valores informales, la visión compartida y el compromiso individual generan que la cultura escolar de VM brinde muestras de un sólido carácter colaborativo que merece ser estudiado en profundidad y de forma independiente.

Hay un ambiente colaborativo. Si hay algo que coordinar, no siento que haya algún inconveniente en compartir responsabilidades, en mencionar qué cosas se tienen que hacer o qué cosas no se tienen que hacer, o qué se hizo mal o qué se hizo bien. Este tipo de sinceridad me parece muy bueno, porque evita los malos entendidos $y$ las suposiciones. Puede haber diferencias, pero "atacan" a la idea, no a la persona. Lo tienen claro. Ninguno grita para exponer una idea; se explica claramente y lo justifican bien. No hay interrupciones ni tampoco es como un salón de clase en el que hay que levantar la mano para intervenir. Estaba implícito eso de que, si alguien hablaba, todos escuchábamos. No habia necesidad de mencionarlo. (Profesor de sexto grado; comunicación personal)

En todo sitio hay una cabeza y los demás estamos orientados a seguir las propuestas que se dan. Cuando creemos que son buenas, apoyamos; cuando creemos que son un poco erradas, tenemos la palabra para poder decirlo, para hacer reconocerle a la dirección que algo está fallando o para cambiary ponernos de acuerdo. (Profesora de cuarto grado, comunicación personal)

Trabajamos estos planes en conjunto. A pesar de que haya jerarquía en la organización, siempre se nos toma en cuenta. A todos los elementos de la organización educativa se nos convoca para trabajar en conjunto. (Profesora de segundo grado; comunicación personal)

Sentia que habia un vínculo muy cercano con los profesores. Éramos muy pocas personas y, por ello, siempre hubo mucha calidez. Yo notaba que ellas se preocupaban mucho por la otra persona y la directora también. Generaban un clima muy cordial, habia mucha tranquilidad. En general, me sentí parte de la institución. Era una comunidad tal cual. (Profesora de computación; comunicación personal)

Basándose en las entrevistas realizadas, podría concluirse que en VM existe, en principio, una cultura de trabajo colaborativo. No obstante, como se dijo previamente, es necesario realizar diversos estudios para sostener tal afirmación, lo cual implica aprehender las diversas concepciones y tratamientos metodológicos del concepto cultura escolar. Además de ello, las culturas escolares no funcionan aisladas del resto de la organización, sino que -esto es lo más importante- dependen de su buen funcionamiento. Considerando que en VM dichas prácticas colaborativas fueron posibles gracias a determinadas políticas, se puede afirmar que una cultura colaborativa puede ser impulsada 
desde el nivel gubernamental, pero esta será realmente desarrollada a nivel directivo y participativo, es decir, en estricta referencia a la escuela y sus miembros. A pesar de las limitaciones de personal e infraestructura, las características organizacionales aquí descritas, sumadas a las nuevas prácticas de gestión escolar, han permitido a VM obtener dos veces consecutivas (2014 y 2015) el Bono Escolar, reconocimiento que otorga el Minedu a aquellas escuelas con altos indicadores de desempeño. De acuerdo con evidencia reciente (Salazar, 2017), dicho recurso constituye uno de los mecanismos destacables de la actual reforma educativa.

\section{Conclusiones y recomendaciones}

La presente investigación discute la aplicabilidad de comunidades profesionales de aprendizaje (CPA) en una escuela pública perteneciente a un sistema educativo de bajo desempeño, mediante el análisis de sus componentes organizativos. Al respecto, se concluye lo siguiente: a) la estructura ejerce una fuerte influencia, al determinar las formas de gestión que desarrollarán las diversas escuelas, por lo que es necesario considerar sus posibilidades y limitaciones en función del contexto sociocultural; b) es importante distribuir el liderazgo mediante comités de gestión en los que los docentes sean responsables del manejo de las instituciones educativas, sin descuidar la posibilidad de implementar instancias semejantes; c) es fundamental compartir valores (enfatizando en la visión y el compromiso individual) en las escuelas, no solo de manera informal, sino también formalmente, a través de normativas; $y$, finalmente, d) es necesario desarrollar culturas escolares colaborativas que sirvan de cimiento a las CPA y que se sostengan en el buen funcionamiento de los demás componentes organizacionales. Son estos cuatro elementos los que deben ser evaluados antes de aplicar cualquier estrategia organizacional y es precisamente la necesidad de tal reflexión previa lo que revela la distancia entre sistemas educativos de alto desempeño y bajo desempeño.
Si buscamos desarrollar CPA (u otras estrategias similares) en sistemas educativos de bajo desempeño, lo más pertinente sería empezar por desarrollar estructuras flexibles (aunque sean generalmente estipuladas por entes rectores alejados de las realidades escolares), liderazgos distribuidos (mediante organismos de gestión docente, como los comités), valores compartidos (formal e informalmente) y culturas escolares colaborativas (que comprendan las dinámicas de las diversas escuelas). Al respecto, se proponen cuatro recomendaciones: a) desarrollar una estructura organizativa flexible que permita la comunicación fluida entre sus niveles y que, para la función directiva, distinga entre gestión y administración; b) promover el liderazgo distribuido y el liderazgo docente mediante comités de gestión o instancias semejantes; c) fomentar valores que dinamicen las relaciones entre pares, no solo de manera informal, sino también mediante normativas, políticas o decálogos; d) impulsar una cultura escolar colaborativa que comprenda la totalidad de agentes de la escuela y constituya el cimiento de su dinámica.

Partiendo del referente empírico empleado y en consideración del análisis realizado, queda por profundizar en la implementación de las comunidades profesionales de aprendizaje. Dado que estas se ejecutan de acuerdo con su influencia en otros elementos organizacionales (estructura, liderazgo, valores y cultura escolar), es responsabilidad del sistema educativo y de la propia escuela velar por su idoneidad. En un principio, la presente investigación tenía por objetivo desarrollar CPA en escuelas públicas de bajo desempeño (a contracorriente de la teoría) para optimizar su rendimiento. Sin embargo, durante la etapa de planificación nos topamos con una realidad distinta que planteó una problemática de necesaria discusión y que puede retratarse a modo de pregunta: ¿cuán efectivas pueden ser determinadas estrategias organizacionales de desempeño académico en escuelas distintas a las referidas? ¿Las diferencias entre sistemas de alto y bajo de desempeño podrían sesgar los resultados obtenidos o atribuirlos a razones equivocadas? Esta proble- 
mática no es solo pertinente en el caso del SEP, sino además en todo sistema educativo que presente características semejantes, especialmente en América Latina (Martins, 2016). Según lo presentado en esta investigación, se justifica una reflexión previa.

$\mathrm{El}$ empleo de estrategias organizacionales no debe ser tomado solo como aquello que la teoría organizacional le brinda a la teoría educativa, sino también como una posible alternativa de reforma. Todos los componentes organizacionales aquí destacados pueden ser hallados en otras escuelas y de maneras diversas, dada la influencia de los contextos socioculturales en los que se ubican (Sai y Siraj, 2015) así como las características de su propio capital humano (Ahn, 2017). Según Cadena-Chala y OrcasitasGarcía, "las comunidades de aprendizaje se consolidan en la medida en que los centros educativos, las instituciones del entorno y los familiares se involucran en el aprendizaje" (2016, p. 390). Al respecto, los cuestionamientos ya han sido señalados, por lo que esta investigación los refiere directamente: "ilas instituciones educativas estarán contribuyendo a generar espacios institucionales en donde fluyan las sinergias entre sus miembros, con el objetivo de que se fomente y genere conocimiento organizacional?" (Álvarez, 2006, p. 16). Únicamente mediante estrategias organizacionales -como las CPA- el SEP podrá cumplir su objetivo principal de "desarrollar la Institución Educativa como comunidad de aprendizaje, encargada de lograr una excelente calidad educativa" (Ministerio de Educación, 2003).

La importancia del presente estudio no descansa únicamente en la reflexión teórico-práctica, sino también en su énfasis en lo organizacional, más allá de lo estrictamente pedagógico, usualmente concebido como el único fin de un sistema educativo. De un tiempo a esta parte, se presume que las soluciones al problema del bajo desempeño educativo deben ejecutarse de arriba hacia abajo (top-down), desde las políticas educativas emitidas por entes gubernamentales hacia las distintas escuelas del territorio nacional. No obstante, aunque dicho trabajo esté mostrando importantes resultados en el SEP (Salazar-Morales, 2017), aquí hemos explorado la tesis inversa: cómo una reforma educativa también podría darse de abajo hacia arriba (bottom-up), desde las muy heterogéneas escuelas hasta las usualmente homogéneas instancias gubernamentales. El flujo de información puede ser bidireccional. Atendiendo a un caso concreto, la presente investigación parte de una perspectiva organizacional que analiza los componentes de una escuela (desde comportamientos, funciones y estructuras hasta normas y valores) en razón de su importancia estratégica para la gestión educativa y que, con base en la evidencia, ha mostrado resultados satisfactorios en los que vale la pena profundizar.

\section{Agradecimientos}

Sincera gratitud a los miembros de la IE Villa Magisterial, de Cerro Colorado (Arequipa), quienes mostraron un destacable compromiso en la ejecución de la presente investigación. Esperamos que este pequeño aporte goce de vuestra aprobación y que, en cierto modo, pueda exponer lo mucho que Uds. han enfrentado y logrado. 


\section{Referencias}

Ahn, J. (2017). Taking a step to identify how to create professional learning communities -Report of a case study of a Korean public high school on how to create and sustain a school-based teacher professional learning community. International Education Series, 10(1), 82-92. https://doi.org/10.5539/ies.v10n1p82

Alayo, F. (2017). Debate por el currículo: Precisiones causaron estas reacciones. El Comercio. https://elcomercio. pe/peru/debate-curriculo-precisiones-causaron-reacciones-143942

Álvarez, G. (2006). El aprendizaje organizacional como eje de desarrollo en la organización escolar. Educación, 15(29), 7-34. http://revistas.pucp.edu.pe/index.php/educacion/article/view/2319

Álvarez, C. y San Fabián, J. (2012). La elección del estudio de caso en investigación educativa. Gazeta de Antropología, 28(1), 1-12. http://www.gazeta-antropologia.es/?p=101

Argote, L. (2011). Organizational learning research: Past, present and future. Management Learning, 42(4), 439446. https://doi.org/10.1177/1350507611408217

Bellibas, M., Bulut, O. y Gedik, S. (2017). Investigating professional learning communities in Turkish schools: The effects of contextual factors. Professional Development in Education, 43(3), 353-374. https://doi.org/10.108 o/19415257.2016.1182937

Beltrán, A. y Seinfeld, J. (2013). La trampa educativa en el Perú: cuando la educación llega a muchos pero sirve a pocos. Lima: UP.

Bolívar, A. (2011). Aprender a liderar líderes: Competencias para un liderazgo directivo que promueva el liderazgo docente. Educar, 47(2), 253-275. https://www.raco.cat/index.php/Educar/article/view/248536

Buttram, J.y Farley-Ripple, E. (2016). The role of principals in professional learning communities. Leadership and Policy in Schools, 15(2), 192-220. https://doi.org/10.1080/15700763.2015.1039136

Cadena-Chala, M. y Orcasitas-García, J. (2016). Comunidades de aprendizaje en el País Vasco: Caracterización y organización escolar. Educación y Educadores, 19(3),373-391. https://doi.org/10.5294/edu.2016.19.3.4

Carpenter, D. (2015). School culture and leadership of professional learning communities. International Journal of Educational Management, 29(5), 682-694. https://doi.org/10.1108/IJEM-04-2014-0046

Castillo, P., Puigdellivol, I. y Antúnez, S. (2017). El liderazgo compartido como factor de sostenibilidad del proyecto de comunidades de aprendizajes. Estudios Pedagógicos, 43(1), 41-59. https://doi.org/10.4067/So71807052017000100003

Chen, P., Lee, C., Lin, H. y Zhang, C. (2016). Factors that develop effective professional learning communities in Taiwan. Asia Pacific Journal of Education, 36(2), 248-265. https://doi.org/10.1080/02188791.2016.1148853

Cheng, X.y Wu, L. (2016). The affordances of teacher professional learning communities: A case study of a Chinese secondary school. Teaching and Teacher Education, 58, 54-67. https://doi.org/10.1016/j.tate.2016.04.008 
ISSN 0123-1294 | e-ISSN 2027-5358 | Educ.Educ. Vol. 23. No. 1 | Febrero-abril de 2020 | pp. 91-112.

Universidad de La Sabana | Facultad de Educación

Cherrington, S. y Thornton, K. (2015). The nature of professional learning communities in New Zealand early childhood education: An exploratory study. Professional Development in Education, 41(2), 310-328. https:// doi.org/10.1080/19415257.2014.986817

Chuquilin, J.y Zagaceta, M. (2017). El currículo de la educación básica en tiempos de transformaciones: Los casos de México y Perú. Revista Mexicana de Investigación Educativa, 22(72), 109-134. http://www.scielo.org.mx/ scielo.php?script=sci_abstract\&pid=S1405-66662017000100109

Consejo Nacional de Educación (2006). Proyecto Educativo Nacional al 2021. Lima: CNE.

Cuenca, R. (2017). Moving toward professional development: The teacher reform in Peru (2012-2016). Lima: IEP.

Deal, T.y Peterson, K. (2016). Shaping school culture. California:Wiley.

Diamond,J.ySpillane,J.(2016).Schoolleadershipand management froma distributed perspective:A 2016 retrospective and prospective. Management in Education, 30(4), 147-154. https://doi.org/10.1177/0892020616665938

Díaz, H. (2015). Formación docente en el Perú. Lima: Santillana.

DiPaola, M. y Hoy, W. (2015). Leadership and school quality. EE.UU.: Information Age Publishing.

Dogan, S., Tatik, R. y Yurtseven, N. (2017). Professional learning communities assessment: Adaptation, internal validity, and multidimensional model testing in Turkish context. Educational Sciences: Theory y Practice, 17(4), 1075-1101. https://eric.ed.gov/?id=EJ1148355

DuFour, R. (2004). What is a professional learning community? Educational Leadership, 61(8), 6-11. http://www. ascd.org/publications/educational-leadership/mayo4/vol61/numo8/What-Is-a-Professional-LearningCommunity\%C2\%A2.aspx

DuFour, R. (2007). Professional learning communities: A bandwagon, an idea worth considering, or our best hope for high levels of learning? Middle School Journal, 39(1), 4-8. https://eric.ed.gov/?id=EJ775771

El Comercio (2017a). Marilú Martens: “Currículo no se está implementando al ritmo propuesto". Recuperado de https://elcomercio.pe/cade/curriculo-implementando-ritmo-propuesto-noticia-478183

El Comercio (2017b). Año escolar 2018: ¿cuándo empezarán clases en colegios públicos? Recuperado de https:// elcomercio.pe/peru/ano-escolar-2018-empezaran-clases-colegios-publicos-noticia-476344

González, L. (2003). El poder de la organización informal en la gestión administrativa. Revista Educación, 27(1), 187-195. https://revistas.ucr.ac.cr/index.php/educacion/article/view/3813

Gray, J., Kruse, S. y Tarter, J. (2015). Enabling school structures, collegial trust and academic emphasis: Antecedents of professional learning communities. Educational Management Administration \& Leadership, 44(6), 875-891. https://doi.org/10.1177/1741143215574505

Guadalupe, C., León, J., Rodríguez, J. y Vargas, S. (2017). Estado de la educación en el Perú: Análisis y perspectivas de la Educación Básica. Lima: Grade, Forge. 
Gutierez, S. (2016). Building a classroom-based professional learning community through lesson study: Insights from elementary school science teachers. Professional Development in Education, 42(5), 801-817. https:// doi.org//10.1080/19415257.2015.1119709

Harris, A. y Jones, M. (2010). Professional learning communities and system improvement. Improving Schools, 13(2), 172-181. https://doi.org/10.1177/1365480210376487

Higgins, K. (2016). An investigation of professional learning communities in North Carolina school systems.Journal of Research/nitiatives,2(1),1-21.https://digitalcommons.uncfsu.edu/cgi/viewcontent.cgi?article=1036\&context=jri

Huffman, J. (2003). The role of shared values and vision in creating professional learning communities. NASSP Bulletin, 87(637), 21-34. https://doi.org/10.1177/019263650308763703

Hunter III, S. (2015). Combining theoretical perspectives on the organizational structure-performance relationship. Journal of Organization Design, 4(2), 24-37. https://doi.org/10.7146/jod.16781

Jopen, G., Gómez, W. y Olivera, H. (2014). Sistema educativo peruano. Lima: PUCP.

Kruse, S. y Johnson, B. (2017). Tempering the normative demands of professional learning communities with the organizational realities of life in schools. Educational Management Administration y Leadership, 45(4), 588-604. https://doi.org/10.1177/1741143216636111

La República (2017). Minedu presentó estrategia para garantizar una educación de calidad en el 2021. Recuperado de http://larepublica.pe/sociedad/1150418-minedu-presento-estrategia-para-garantizar-una-educacion-de-calidad-en-el-2021

Lai, E. y Cheung, D. (2015). Enacting teacher leadership: the role of teachers in bringing about change. Educational Management Administration \& Leadership, 43(5), 673-692. https://doi.org/10.1177/1741143214535742

Lam, Y. (2005). School organizational structures: Effects on teacher and student learning. Journal of Educational Administration, 43(4), 387-401. https://doi.org/10.1108/09578230510605432

Lee, M. y Kim, J. (2016). The emerging landscape of school-based professional learning communities in South Korean schools. Asia Pacific Journal of Education, 36(2), 266-284. https://doi.org/10.1080/02188791.2016.1148854

Leithwood, K. (2009). ¿Cómo liderar nuestras escuelas? Santiago: Área de Educación Fundación Chile.

Martins, A. (2016). Pruebas PISA: ¿Cuáles son los países que tienen la mejor educación del mundo? ¿Y cómo se ubica América Latina? BBC Mundo. http://www.bbc.com/mundo/noticias-38211248

Ministerio de Educación (2003). Ley General de Educación. Lima: Minedu.

Ministerio de Educación (2012). Marco de buen desempeño docente. Lima: Minedu.

Ministerio de Educación (2013). La gestión descentralizada de la educación. Lima: Minedu. 
ISSN 0123-1294 | e-ISSN 2027-5358 | Educ.Educ. Vol. 23. No.1 | Febrero-abril de 2020 | pp. 91-112.

Universidad de La Sabana | Facultad de Educación

Ministerio de Educación (2015a). Marco del buen desempeño del directivo. Lima: Minedu.

Ministerio de Educación (2015b). Manual de gestión escolar. Lima: Minedu.

Ministerio de Educación (2016a). El impulso de una carrera: política de revalorización docente en el Perú. Lima: Minedu.

Ministerio de Educación (2016b). Semáforo escuela: manual del monitor. Lima: Minedu.

Ministerio de Educación (2017). Currículo Nacional de la Educación Básica. Lima: Minedu.

Morales, S. y Morales, O. (2018). Comunidades profesionales de aprendizaje: De la gestión empresarial a la gestión educativa. Revista Peruana de Investigación Educativa, 10, 99-125. https://revistas.siep.org.pe/index. $\mathrm{php/RPIE/article/view/89}$

Moreano, G. (coord.) (2017). El Perú en PISA 2015. Informe nacional de resultados. Lima: Minedu.

Mulford, B.y Silins, H. (2010). Organizational learning in schools. En P. Peterson, E. Baker y B. McGaw (eds.), International encyclopedia of education (pp. 142-150). Oxford: Elsevier.

OCDE - Organización para la Cooperación y el Desarrollo Económicos (2016). Avanzando hacia una mejor educación para Perú. Lima: UP.

Olivier, D. y Huffman, J. (2016). Professional learning community process in the United States. Asia Pacific Journal of Education, 32(2), 301-317. https://doi.org/10.1080/02188791.2016.1148856

Pang, N. y Wang, T. (2016). Professional learning communities: Research and practices across six educational systems in the Asia-Pacific region. Asia Pacific Journal of Education, 36(2), 193-201. https://doi.org/10.1080 /02188791.2016.1148848

Pang, N., Wang, T.y Leung, Z. (2016). Educational reforms and the practices of professional learning community in Hong Kong primary schools. Asia Pacific Journal of Education, 36(2), 231-247. https://doi.org/10.1080/02 188791.2016.1148852

Peterson, K. y Deal, T. (2009). The shaping school culture fieldbook. San Francisco: Wiley.

Pirela, L. y Sánchez, M. (2009). Cultura y aprendizaje organizacional en instituciones de Educación Básica. Revista de Ciencias Sociales, 15(1), 175-188. http://ve.scielo.org/scielo.php?pid=S1315-95182009000100013\&script=sci_arttext

Popp, J.y Goldman, S. (2016). Knowledge building in teacher professional learning communities: Focus of meeting matters. Teaching and Teacher Education, 59, 347-359. https://doi.org/10.1016/j.tate.2016.06.007

Qiao, X., Yu, S. y Zhang, L. (2018). A review of research on professional learning communities in mainland China (2006-2015). Educational Management Administration \& Leadership, 46(5), 713-728. https://doi. org/10.1177/1741143217707523 
Rule, P. y John, V. (2015). A necessary dialogue: Theory in case study research. International Journal of Qualitative Methods, 14(4), 1-11. https://doi.org/10.1177/1609406915611575

Rutherford, C. (2006). Teacher leadership and organizational structure: The implications of restructured leadership in an Edison school. Journal of Educational Change, 7(1-2), 59-76. https://doi.org/10.1007/s10833oo6-0013-4

Sai, X. y Siraj, S. (2015). Professional learning community in education: Literature review. The Online Journal of Quality in Higher Education, 2(2), 65-78. https://www.tojqih.net/journals/tojqih/articles/vo2ioz/

Salazar-Morales, D. (2018) Sermons, carrots or sticks? Explaining successful policy implementation in a low performance institution. Journal of Education Policy, 33(4), 457-487. http://doi.org/10.1080/02680939.2017.1378823

Salleh, H. (2016). Facilitation for professional learning community conversations in Singapore. Asia Pacific Journal of Education, 36(2), 285-300. https://doi.org/10.1080/02188791.2016.1148855

Sandín, M. (2003). Investigación cualitativa en educación. Madrid: McGraw-Hill.

Sigurðardóttir, A. (2010). Professional learning community in relation to school effectiveness. Scandinavian Journal of Educational Research, 54(5), 395-412. https://doi.org/10.1080/00313831.2010.508904

Sjoer, E.y Meirink, J. (2016). Understanding the complexity of teacher interaction in a teacher professional learning community. European Journal of Teacher Education, 39(1), 110-125. https://doi.org/10.1080/02619768 .2014 .994058

Sutton, P.y Shouse, A. (2016). Building a culture of collaboration in schools. Phi Delta Kappan, 97(7), 69-73. https:// doi.org/10.1177/0031721716641653

Svanbjörnsdóttir, B., Macdonald, A. y Frímannsson, G. (2016a). Views of learning and a sense of community among students, paraprofessionals and parents in developing a school culture towards a professional learning community. Professional Development in Education, 42(4), 589-609. https://doi.org/10.1080/194 15257.2015 .1047037

Svanbjörnsdóttir, B., Macdonald, A. y Frímannsson, G. (2016b). Teamwork in establishing a professional learning community in a new Icelandic school. Scandinavian Journal of Educational Research, 60(1), 90-109. https://doi.org/10.1080/00313831.2014.996595

Tam, A. (2015a). The role of a professional learning community in teacher change: A perspective from beliefs and practices. Teachers and Teaching, 21(1), 22-43. https://doi.org/10.1080/13540602.2014.928122

Tam, A. (2015b). Exploring teachers' beliefs about teacher learning in professional learning communities and their influence on collegial activities in two departments. Compare, 45(3), 422-444. https://doi.org/10.108 o/03057925.2013.872025 
Vanblaere, B. y Devos, G. (2016). Relating school leadership to perceived professional learning community characteristics: A multilevel analysis. Teaching and Teacher Education, 57, 26-38. https://doi.org/10.1016/j. tate.2016.03.003

Vanblaere, B. y Devos, G. (2018). The role of departmental leadership for professional learning communities. Educational Administration Quarterly, 54(1), 85-114. https://doi.org/10.1177/0013161X17718023

Vescio, V., Ross, D. y Adams, A. (2008). A review of research on the impact of professional learning communities on teaching practice and student learning. Teaching and Teacher Education, 24(1), 80-91. https://doi. org/10.1016/j.tate.2007.01.004

Villalobos, M. (2017). ¿Cuál es la situación de los colegios privados en el Perú? El Comercio. https://elcomercio.pe/ economia/negocios/situacion-colegios-privados-peru-162820

Wang, T. (2016). School leadership and professional learning community: Case study of two senior high schools in Northeast China. Asia Pacific Journal of Education, 36(2), 202-216. https://doi.org/10.1080/02188791.201 6.1148849

Watson, C. (2014). Effective professional learning communities? The possibilities for teachers as agents of change in schools. British Educational Research Journal, 40(1), 18-29. https://doi.org/10.1002/berj.3025

Wennergren, A. y Blossing, U. (2017). Teachers and students together in a professional learning community. Scandinavian Journal of Educational Research, 61(1), 47-59. https://doi.org/10.1080/00313831.2015.1066441

Wong, J. (2010). What makes a professional learning community possible? A case study of a Mathematics department in a junior secondary school of China. Asia Pacific Education Review, 11(2), 131-139. https://doi. org/10.1007/s12564-010-9080-6

Woods, P. (1987). La escuela por dentro: La etnografia en la investigación educativa. Barcelona: Paidós.

Yon, D. (2003). Highlights and overview of the history of educational ethnography. Annual Review of Anthropology, 32, 441-429. https://doi.org/10.1146/annurev.anthro.32.061002.093449

Zhang, J. y Pang, N. (2016). Exploring the characteristics of professional learning communities in China: A mixedmethod study. The Asia-Pacific Education Researcher, 25(1), 11-21. https://doi.org/10.1007/s40299-015-0228-3

Zhang, J., Yuan, R. y Yu, S. (2017). What impedes the development of professional learning communities in China? Perceptions from leaders and frontline teachers in three schools in Shanghai. Educational Management Administration \& Leadership, 45(2), 219-237. https://doi.org/10.1177/1741143215617945 